\section{Heart Involvement in a Woman Treated with Hydroxychloroquine for Systemic Lupus Erythematosus Revealing Fabry Disease}

\section{To the Editor:}

Chloroquine (CQ) and hydroxychloroquine (HCQ) are widely used in the longterm treatment of connective tissue disease (CTD) and are usually considered safe ${ }^{1}$. However, these therapies may cause serious adverse events ${ }^{2}$, including cardiac toxicity ${ }^{3}$. The cardiomyopathy induced by an antimalarial drug might mimic the cardiac involvement of Fabry disease (FD), a genetic storage disorder that causes a deficiency of a lysosomal enzyme, alpha galactosidase A (GLA). Indeed, CQ and HCQ provoke a dysfunction in the lysosomal enzymes, leading to the impairment of intracellular degradation processes in conjunction with the accumulation of pathological metabolic products ${ }^{4,5}$. The cardiac presentation of these 2 diseases is close regarding the clinical symptoms as well as the imaging and pathological findings. We report the case of a patient, treated longterm with HCQ, with cardiac disorders revealing FD.

A 61-year-old woman was diagnosed 20 years ago for a CTD with dermatological disorders: alopecia, skin rash, and joint pain. The antinuclear antibodies (ANA) were positive at 1/100. Anti-SSa/Ro and anti-dsDNA antibodies were negative. The skin biopsy showed moderate lymphocytic infiltrate. Systemic lupus erythematosus (SLE) was diagnosed and HCQ (200 mg twice daily) was started in combination with low doses of prednisone (10 mg per day), which led to clinical improvement. Seventeen years later, she presented sudden neurologic disorders with dizziness, left arm weakness, and facial paralysis. The brain magnetic resonance imaging (MRI) showed a recent ischemic stroke of the right middle cerebral artery. The physical examination did not find high blood pressure, cardiac arrhythmia, or stenosis of upper-aortic vessels. The echocardiogram showed left ventricular hypertrophy without embolic disorder. Therapy with aspirin, $\beta$-blockers, and angiotensin-converting enzyme was started. One month later, she developed persistent asthenia and chest pain with dyspnea. The electrocardiogram showed normal sinus rhythm without repolarization abnormalities. The highly sensitive troponin was elevated to $110 \mathrm{ng} / \mathrm{l}$ (normal below $14 \mathrm{ng} / \mathrm{l}$ ). Coronary artery angiogram was normal. Contrast-enhanced heart MRI showed a hyperintense T2 signal in the epicardial zone with delayed contrast enhancement. Echocardiography revealed concentric left ventricular hypertrophy and normal systolic function. ANA as well as antiphospholipid syndrome antibodies were negative. Initially, myocarditis was retained and a corticosteroid infusion treatment was initiated. This therapy led to an improvement in the clinical symptoms, but with persistent high levels of troponin. A few days later, endomyocardial biopsies were performed and detected severe cytoplasmic vacuolization of cardiomyocytes by light microscopy without signs of vasculitis or amyloidosis (Figure 1A). Transmission electron microscopy revealed many myelin figure inclusions, few curvilinear bodies, and glycogen accumulation in cardiomyocytes (Figure 1B). Myelin figures were also present in vascular smooth muscle cells and fibroblasts. The dosage of GLA activity was low, at $2 \mu \mathrm{kat} / \mathrm{kg}$ protein (normal range $10-19 \mu \mathrm{kat} / \mathrm{kg}$ protein). The mutation p.Phe113Leu of the GLA gene for FD was present in a heterozygous form. Renal function was impaired with a Modification of Diet in Renal Disease Study equation glomerular filtration rate at $50 \mathrm{ml} / \mathrm{min} / 1.73 \mathrm{~m}^{2}$ without proteinuria. In addition, we retrospectively found that 2 of the patient's brothers were dead of an early sudden cardiac ischemia while under 50 years old. Our definitive diagnosis was cardiomyopathy attributable to FD, probably promoted by the longterm use of HCQ (total cumulative dose of $2480 \mathrm{~g})$.

FD is associated with cardiovascular disorders (myocardial ischemia and brain strokes) and renal impairment. Only $70 \%$ of women with the mutation of the GLA gene have manifestations of $\mathrm{FD}^{5}$. A large number of women are not diagnosed ${ }^{6,7}$. The pathology of myocardial tissue is extremely close between FD and HCQ toxicity. In their study, Roos, et $a l^{8}$ compared both cardiac disorders. They observed the same clinical presentation and the same pathological features with myelin figures and glycogen accumulation in both biopsies. However, they concluded that curvilinear bodies were only present in CQ toxicity ${ }^{8}$. Here, some curvilinear bodies were described, likely meaning that HCQ is partially involved in the cardiac failure. In the literature, to our knowledge, 23 patients with HCQ or CQ cardiotoxicity are reported. They were frequently women and the duration of treatment was between 9 and 35 years. Regarding heart presentation, almost all the patients had left ventricular hypertrophy. The other cardiac events were conduction disorders, rhythm disorders, congestive heart failure, ischemia with hypokinesis, and diastolic dysfunction. Several patients presented chest pain, increasing dyspnea, and elevated cardiac enzymes. Treatment withdrawal could have beneficial effects in some patients with recovery of heart function, but sometimes the development was unfavorable with irreversible damage, death, or heart transplant. FD was screened in only 7 patients (6 negative and 1 already known positive), whereas the pathologic features could correspond to FD. Only 1 case reported on SLE treated with HCQ
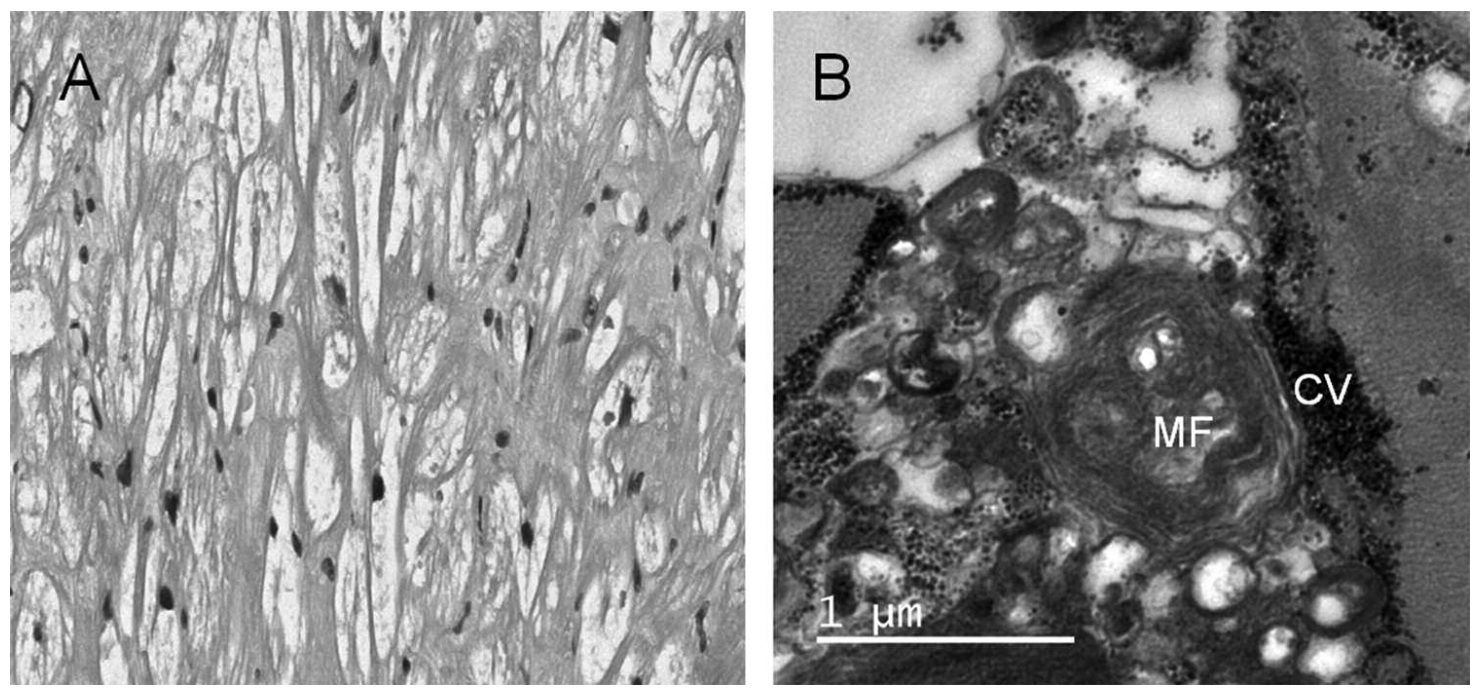

Figure 1. A. Light microscopy image of an endomyocardial biopsy in H\&E stain demonstrates abnormal vacuoles in cardiomyocytes (magnification 40x). B. Transmission electron microscopy: MF inclusion and CV bodies in cardiomyocytes (magnification 4000x). MF: myelin figures; $\mathrm{CV}$ : curvilinear.

Personal non-commercial use only. The Journal of Rheumatology Copyright @ 2016 . All rights reserved. 
associated with $\mathrm{FD}^{9}$. The endomyocardial biopsy showed only myelin figures. Despite HCQ withdrawal and enzyme replacement therapy, this patient continued to have vascular complication with a myocardial infarction and stroke ${ }^{10}$.

Our case highlights HCQ cardiotoxicity and the risk of misdiagnosed FD, especially in women because of nonspecific symptoms. We must also point out the need to realize cardiac biopsies in atypical cardiac involvement in SLE.

CLOTILDE CHATRE, MD, Clinical Immunology and Osteoarticular Diseases Therapeutic Unit, Lapeyronie University Hospital; NATHALIE FILIPPI, MD, Clinical Immunology and Osteoarticular Diseases Therapeutic Unit, Lapeyronie University Hospital, Montpellier, France; FRANÇOIS ROUBILLE, MD, PhD, Cardiology, Arnaud de Villeneuve University Hospital, Montpellier, France, and PhyMedExp, University of Montpellier, INSERM U1046, CNRS UMR 9214, Montpellier, France; YVES-MARIE PERS, MD, Clinical Immunology and Osteoarticular Diseases Therapeutic Unit, Lapeyronie University Hospital, Montpellier, France. Address correspondence to Dr. Y.M. Pers, 371 Avenue du Doyen Gaston Giraud, 34295 Montpellier, France. E-mail: ympers2000@yahoo.fr

\section{REFERENCES}

1. Costedoat-Chalumeau N, Leroux G, Amoura Z, Piette JC. [Hydroxychloroquine and systemic lupus: a reappraisal]. [Article in French] Rev Med Interne 2008;29:735-7.

2. Tönnesmann E, Kandolf R, Lewalter T. Chloroquine cardiomyopathy - a review of the literature. Immunopharmacol Immunotoxicol 2013;35:434-42.

3. Yogasundaram H, Putko BN, Tien J, Paterson DI, Cujec B, Ringrose $\mathrm{J}$, et al. Hydroxychloroquine-induced cardiomyopathy: case report, pathophysiology, diagnosis, and treatment. Can J Cardiol 2014;30:1706-15.
4. Putko BN, Wen K, Thompson RB, Mullen J, Shanks M, Yogasundaram H, et al. Anderson-Fabry cardiomyopathy: prevalence, pathophysiology, diagnosis and treatment. Heart Fail Rev 2015;20:179-91.

5. Sheppard MN. The heart in Fabry's disease. Cardiovasc Pathol 2011;20:8-14.

6. Wilcox WR, Oliveira JP, Hopkin RJ, Ortiz A, Banikazemi M, Feldt-Rasmussen U, et al; Fabry Registry. Females with Fabry disease frequently have major organ involvement: lessons from the Fabry Registry. Mol Genet Metab 2008;93:112-28.

7. Laaksonen SM, Röyttä M, Jääskeläinen SK, Kantola I, Penttinen M, Falck B. Neuropathic symptoms and findings in women with Fabry disease. Clin Neurophysiol 2008;119:1365-72.

8. Roos JM, Aubry MC, Edwards WD. Chloroquine cardiotoxicity: clinicopathologic features in three patients and comparison with three patients with Fabry disease. Cardiovasc Pathol 2002; 11:277-83.

9. Nandagudi A, Jury EC, Alonzi D, Butters TD, Hughes S, Isenberg DA. Heart failure in a woman with SLE, anti-phospholipid syndrome and Fabry's disease. Lupus 2013;22:1070-6.

10. Mehta A, Beck M, Elliott P, Giugliani R, Linhart A, Sunder-Plassmann G, et al; Fabry Outcome Survey investigators. Enzyme replacement therapy with agalsidase alfa in patients with Fabry's disease: an analysis of registry data. Lancet 2009; 374:1986-96.

J Rheumatol 2016;43:5; doi:10.3899/jrheum.151357 